Cahiers de recherches médiévales

\title{
Le compagnon sans mémoire
}

Le siège d'Orléans dans le Jouvencel de Jean de Bueil

\section{Françoise Michaud-Fréjaville}

\section{CpenEdition}

\section{Journals}

Édition électronique

URL : https://journals.openedition.org/crm/725

DOI : $10.4000 / \mathrm{crm} .725$

ISSN : 1955-2424

Éditeur

Honoré Champion

\section{Édition imprimée}

Date de publication : 1 juin 2005

Pagination : 101-111

ISSN : $1272-9752$

Référence électronique

Françoise Michaud-Fréjaville, "Le compagnon sans mémoire », Cahiers de recherches médiévales [En ligne], 12 spécial | 2005, mis en ligne le 28 juin 2008, consulté le 15 décembre 2022. URL : http:// journals.openedition.org/crm/725; DOI : https://doi.org/10.4000/crm.725 


\title{
rin
}

\author{
$-9-$ \\ Le compagnon sans mémoire \\ Le siège d'Orléans dans le Jouvencel de Jean de Bueil'
}

«La plupart des chouses qui sont escrites oudit livre du Jouvencel, il les a faites et executees dont d'autres s'en sont donné la gloire de les avoir faictes », dit de Jean de Bueil un de ses serviteurs, Guillaume Tringant, qui avait assisté à la genèse et à la rédaction de l'ouvrage. Cette modestie de l'amiral, nous pouvons la discuter en notant que sa mémoire fut parfois sélective, et que la transmutation des souvenirs en roman s'est accompagnée de quelques fâcheuses lacunes. Ces absences se sont non seulement traduites dans son récit mais aussi dans quelques prises de positions politiques - ses participations à la Praguerie et à la guerre du Bien Public sont en contradiction avec ses discours sur la fidélité au Roi - et dans son abstention lors du procès en nullité de la condamnation de Jeanne d' $\mathrm{Arc}^{2}$. C'est cette distorsion entre les souvenirs embellis ou réduits, de toute façon réécrits, et la réalité historique que je tenterai d'éclairer un peu ici.

Dans Le Jouvencel, récit autobiographique à peine déguisé, dont les clefs ont été presque immédiatement données par Tringant ${ }^{3}$, l'amiral, déjà âgé - il a une soixantaine d'années - évoque sa jeunesse, sa carrière, ses compagnons d'armes ; il rend hommage en particulier à Dunois, le bâtard d'Orléans, et Jean, duc d'Alençon dont il fait dans son texte un seul personnage, sous le nom du comte de Parvenchè-

\footnotetext{
${ }^{1}$ Une première version de cette étude a été proposée au séminaire de J. Cerquiglini, «L'écriture aux $X I V^{\mathrm{e}}$ et $X V^{\mathrm{e}}$ siècles, étude sémiotique et sociologique».

${ }^{2}$ Rappelons brièvement la carrière de Jean V de Bueil, fils de Jean IV, mort à Azincourt, et de Marguerite Dauphine. Il naît en 1406. Son enfance est relativement pauvre et difficile. Devenu page, il assiste aux journées de Cravant (1423) et de Verneuil (1424). Il suit ensuite Etienne de Vignolles dit La Hire à Orléans (1428-1429), puis continue comme chef de compagnie. Il est dit chevalier en 1434. Capitaine de l'Ordonnance en 1445, il participe à la reconquête de la Normandie, est fait amiral de France en 1450, hérite du comté de Sancerre en 1451, combat à Castillon (1453) et reçoit la reddition de Bordeaux. Tombé en disgrâce après la mort de Charles VII, il se retire au château de Vaujours et commence à raconter le Jouven$\mathrm{cel}$ que trois collaborateurs notent et rédigent. Brièvement revenu à la vie publique en 1465 pour suivre Charles, le frère du roi, dans sa révolte «pour le Bien Public» contre Louis XI, il fait soumission et retourne à sa retraite. Il meurt à Vaujours au début de l'été 1477. Il est enterré dans la collégiale de Bueil. Son fils, Antoine, avait épousé en 1461 Jeanne, troisième fille de Charles VII et d'Agnès Sorel.

${ }^{3}$ Le Jouvencel par Jean de Bueil, suivi du commentaire de Guillaume Tringant, introduction par C. Favre, texte établi par L. Lecestre, Paris, Société de l'Histoire de France, 1887, 2 vol. Dans son introduction, C. Favre indique que le commentaire, ajouté d'une autre main au roman dans le manuscrit de l'Arsenal qui sert de référence à l'édition, était « déjà transcrit sur certains manuscrits avant la fin du $\mathrm{XV}^{\mathrm{e}}$ siècle» (Introduction biographique, t. I, p. CCCXXVII).
}

Cahiers de Recherches Médiévales (XII $-X V^{e}$ s.), 12spé, 2005 
$\mathrm{res}^{4}$. On peut alors se demander pourquoi, parvenu à un âge de sagesse, couvert de gloire après les campagnes menées pour Charles VII le «Victorieux » et n'ayant pas craint de manifester au cours de son existence quelques capacités d'indépendance d'esprit, il n'a pas fait entrer dans son récit une «preuse » qui eût ajouté encore du romanesque et fait revenir en mémoire aux lecteurs l'histoire de la Pucelle d'Orléans. Plus intimement, la lecture de cette évocation tronquée permettra peutêtre de comprendre les raisons pour lesquelles il n'a pas associé en 1456 son témoignage lors des enquêtes de réhabilitation ${ }^{5}$ à ceux de Dunois, de Jean d'Alençon et de Raoul de Gaucourt, ses maîtres en matière militaire, les éducateurs de sa jeunesse armée et aventureuse ${ }^{6}$.

Au chapitre XI du livre II du Jouvencel se déroule le siège de Crathor, pseudonyme d'un certain nombre de cités assiégées dont le héros aida à la délivrance. Le commentateur du $\mathrm{XV}^{\mathrm{e}}$ siècle nous dit: «[...] et pour vous parler plus clairement de Crator, il est prins pour le siège d'Orléans et de Lagny-sur-Marne et après nommèrent-ils Crator le chasteau de Sablé $»^{7}$. Il ne me semble pas pourtant que l'on puisse s'attarder outre mesure sur un éventuel récit concernant Lagny: Jean de Bueil n'a pas assisté à cet épisode de la guerre autour de Paris (août 1432), il était alors en train de dégager de la pression anglaise la petite cité de Saint-Céneri dans l'Orne ${ }^{8}$. Donc, le héros, après avoir pris une ville nommée Escallon (ici Marchenoir), se trouve à son tour assiégé dans Crathor par des ennemis dont le chef est le duc de Baudoyn, nom collectif pour tous les lieutenants du roi d'Angleterre', et c'est le récit de la délivrance de la cité par les troupes envoyées par le roi qui sera analysé.

${ }^{4}$ Les Lieutenants du roi sont désignés génériquement par ce nom : «Ce eust été forte chouse d'avoir couché [par écrit] tous les Lieutenants que le roi ordonnoit ; car il en a eu plusieurs et en plusieurs pays et contrees. Entre les autres, il s'est fort aydé du conte de Dunois » ( $L e$ Jouvencel, t. II, Commentaire, XXXVII, p. 298). «Le Jouvencel fut lieutenant du conte du Mayne (Charles d'Anjou) et du duc d'Alençon quelquefoys. Et pour ce est-il dit en son livre qu'il estoit lieutenant du conte de Parvencheres. Et pour mieulx deguiser toujours icelluy livre, on les appelle tous le conte de Parvencheres » (ibid., XXVIII, p. 299).

${ }^{5}$ Le nom de Jean de Bueil est en fait totalement absent du procès en nullité de la condamnation de Jeanne d'Arc: cf. P. Duparc, Procès en nullité de la condamnation de Jeanne d'Arc, Paris, Klincsieck (Société d'Histoire de France), 1988, t. V, Index I, p. 227-249.

${ }^{6}$ Notons, à la décharge de notre auteur, que Poton de Xaintrailles (†1461) n'a pas non plus été entendu lors du procès en nullité. La Hire et Gilles de Rais étaient morts.

${ }^{7}$ Le Jouvencel, Commentaire de Tringant, t. II, XVII, p. 283.

${ }^{8}$ Lagny fut délivrée pratiquement sans livrer bataille, par un double mouvement de troupes : pendant qu'une partie des Français, menés par Jean, Bâtard d'Orléans, et Raoul, de Gaucourt, réussissaient à entrer dans la ville par un point mal surveillé, Gilles de Rais et Rodrigue de Villandrando traversaient la Marne en amont, avant la Ferté-sous-Jouarre. Devant la menace, les Anglais abandonnèrent les bastilles et le pont qu'ils tenaient en aval de Lagny, laissant sur place une bonne partie de leur équipement. Cf. le récit, pas toujours très sûr, de Jean Chartier, Chronique, éd. Vallet de Viriville, Paris, 1858, t. I, p. 143-146). L'annotateur du Jouvencel, L. Lecestre, dans une longue note, essaie de distinguer Orléans de Lagny dans le récit (t. I, p. 220, n. 1 et p. 221) : ce n'est pas très convaincant.

${ }^{9}$ Le Jouvencel, Commentaire de Tringant, t. II, p. 298, XXXVIII. 


\section{Jean de Bueil à Orléans}

Avant de comparer le récit de l'amiral avec ce que nous savons de l'affaire d'Orléans, il faut rappeler quelle part il prit aux secours apportés à la ville assiégée. Nous savons par les comptes royaux qu'il vint avec Dunois dès le mois d'octobre 1428 , menant avec lui quinze hommes d'armes ${ }^{10}$, ce que reprend la Chronique de la Pucelle $^{11}$. Il a alors 22 ans environ. Selon Guillaume Tringant, il serait resté avec Dunois quelque temps dans Orléans après la journée des Harengs ${ }^{12}$. Nous le retrouvons le 3 mai 1429, avec le ravitaillement venu de Blois et davantage d'hommes sous ses ordres ${ }^{13}$. Enfin, il prit part à la levée du siège, à la campagne de recouvrement de Janville, Jargeau, Meung, Beaugency et au voyage de Reims ${ }^{14}$. On ne peut guère douter des renseignements de première main que pourrait contenir le roman sur les opérations militaires et sur la mentalité des spécialistes de la guerre qui participèrent à la lutte contre les Anglais qui tenaient la rive sud de la Loire autour

${ }^{10}$ Jules Loiseleur, «Compte des dépenses faites par Charles VII pour secourir Orléans pendant le siège de 1428 », Mémoires de la Société Archéologique et Historique de l'Orléanais, t. XI, 1868, p. 169 : Mandement en vertu des lettres patentes données à Chinon le 30 octobre 1428 : «Aux capitaines et chiefs de guerre ci après nommez [...] pour le payement d'eulx et des gens d'armes et de traict de leur compaignie et pour les estats de leur personnes, lesquels estoient allez et entrez en la ville d'Orleans pour y servir ledit seigneur a l'encontre des Anglois, ses anciens ennemis et adversaires y tenans le siege de l'un des costez [...]. A M. de Bueil, quinze hommes d'armes, CLV 1. t. ».

${ }^{11}$ Chronique de la Pucelle ou Chronique de Cousinot, éd. Vallet de Viriville, Paris, 1869, p. 263 : «Mais le bastard d'Orléans, La Hire, Monseigneur de Bueil, Monseigneur de Chaumont $[. .$.$] vinrent le lundi aprés la susdite prise à grand compaignie de gens d'armes ».$

${ }^{12}$ Le Jouvencel, Commentaire de Tringant, t. II, p. 277, X: Après la sanglante défaite de Patay, «le surplus s'en retourna à Orléans et de là s'en allèrent le duc de Borbon, le viconte de Thouars [...] et ne demoura que la garnison accoustumée, dont mon maître fust ung ».

${ }^{13}$ Chronique de la Pucelle, op. cit., p. 287, «Et le même jour ou soir (du 3 mai) vinrent nouvelles que le Maréchal de Sainte Sévère, le sire de Rays, Monseigneur de Bueil et La Hire, qui amenoient les vivres et l'artillerie». Cela est confirmé par les livres de comptes d'Hémon Raguier : (sommes payées en avril et mai 1429) «pour deffrayer eulx et leurs gens des grandes charges et depenses que faire leur avoit convenu [...] qui avoient mené et conduit en la seconde fois les vivres [...] en la ville d'Orléans pour l'advitaillement et fortification [...] à Monseigneur Jehan, sire de Bueil, chevalier, sur le paiement de XXX hommes d'armes et XL hommes de traict, IIc 1.t». On notera que la mise au net de ce compte fait de Jean de Bueil un chevalier, alors qu'il ne semble avoir porté ce titre qu'en 1434.

${ }^{14}$ J. Loiseleur, op. cit., p. 199, répartition de 17434 1.t.. entre les capitaines qui ont participé «au lievement du siège, $[\ldots]$ en la recouvrance des villes et forteresses [...] où voyage et assemblée par icelluy seigneur (roi) faite à Reims pour le faict de son sacre et couronnement [...]. À Mons. de Bueil, IIc 1.t. ». Enfin, Loiseleur signale, dans un relevé fait par Lottin (Médiathèque d'Orléans, ms. 449 bis) des comptes de Jean Hilaire, receveur de la ville en 14291431, un paiement fait en vertu de l'autorisation des procureurs de ville en date du 14 octobre 1429. Ce mandement reproduit une partie du mandement ci-dessus d'Hémon Raguier et, en particulier, donne exactement la même liste des bénéficiaires dans le même ordre; mais le copiste a lu en quinzième position « $\mathrm{M}$. Dubreuil» au lieu de « $\mathrm{M}$. de Bueil ». Cette erreur, qui n'est pas la seule (Dalebret est lu d'Albert, Raiz devient Ryeux) montre que le nom de Bueil n'est pas familier aux Orléanais (Loiseleur, op. cit. p. 208). 
d'Orléans en 1428-1429. L'intérêt est de chercher à percer la transmutation romanesque.

\section{Le siège dans le Jouvencel}

Dans le roman, l'affaire commence le 8 août (ce qui est davantage conforme, il faut le reconnaître, aux péripéties du blocage de Lagny par les troupes du duc de Bedford) : «Les ennemis se logerent en trois lieux devant la place, non pas si près que le canon leur peust faire guerre. Et au milieu de leurs trois sièges firent une fortification près de la ville, là où leur sembla estre plus guerroiable, tant de bombardes que d'approches» (p. 164). «[...] Et si peuvent bien de leur champs secourir leur guet et leur artillerie par le moyen d'un long fossé qu'ils ont fait pour aller de l'un à l'autre » (p. 169). Nous savons que les Anglais s'installèrent sur la rive sud de la Loire, en trois points : le Champ-Saint-Privé, les Augustins et Saint-Jean-le-Blanc. Le texte ne parle pas ici du fleuve ni du pont, mais il en est question un peu plus loin quand on conseille aux secours de traverser la rivière de nuit, celle-ci s'appelle alors la Faire (p. 176), et quand il s'avère nécessaire de bien protéger les renforts entrés dans la ville : «néanmoins qu'il y avoit une rivière entre eulx et leurs ennemis. Mais il y avoit un pont, qui estoit juste encontre le champ de leurs ennemyz, et en estoient seigneurs les adversaires et povoient passez quant il leur plaisoit, néanmoins avoient ung gué à leur commandement au dessoubz du pont $»^{15}$. Le «gué » en question est celui de l'île Charlemagne, où les Anglais avaient installé une bastille qui interdisait aux assiégés la sortie par le fleuve vers l'amont. Il est évident que le Jouvencel est assez gêné pour expliquer ce double passage sur l'eau. En fait, le pont d'Orléans était pour les ennemis en cul-de-sac vers la ville, immédiatement après le fort des Tourelles, puisque, le 22 octobre 1428, on avait fait sauter l'arche précédant la Belle Croix. C'est à Lagny qu'ils avaient la maitrise du pont, mais alors ils n'avaient pas de gué. On voit comment fonctionnent ici le souvenir, l'intégration des récits recueillis au cours des campagnes militaires, la dissimulation volontaire et l'écriture: par accumulation et non par sélection, ce qui rend les justifications nécessaires et embarrassées.

Très vite les bombardements commencent: «Ils descendirent leur grosse artillerie et commencèrent fort à battre $»^{16}$. Nous savons que, depuis la levée de SaintJean-le-Blanc, trois grosses pièces firent feu dès le dimanche 17 octobre $1428^{17}$ et que les défenseurs durent, au bout de dix jours, se résoudre à subir un siège. Dans le roman au contraire, et malgré la mitraille, l'installation de Baudoyn, chez des assiégeants, et de ses hommes ne se fait que péniblement : il fallut «plus d'un mois avant qu'ils peussent tenir à leurs intentions ». Mais alors l'encerclement se perfectionne, des contre-fortifications sont creusées, réunies par des tranchées, l'artillerie y est installée « et de là pensoient battre la ville».

\footnotetext{
${ }^{15}$ Le Jouvencel, I, p. 194.

${ }^{16}$ Ibid., p. 164.

${ }^{17}$ « Jettèrent les Anglais dedans la cité cent vingt-quatre pieces de bombardes avec gros canons, dont telle pierre y avait qui pesoit cent seize livres », (Journal du siège, cité dans R. Pernoud, La Libération d'Orléans, Paris,1969, p. 83).
} 
Les défenseurs de Crathor-Orléans ne restent pas impassibles : le Jouvencel, chef des assiégés, a fait deux ou trois sorties : «Et y eust de moult belles apertises d'armes faittes. Touttefois la faulx paist le pré. Et, car ils <les ennemis> estoient moult grant puissance devant, en la fin ils mirent tout leur effort et firent tant [...] que le Jouvencel et ses compaignons se sentirent fort pressez, et tindrent conseil et avisèrent qu'il estoit de nécessité envoyer devers le prince quérir secours $»^{18}$. Effectivement, malgré les sorties des 30 décembre 1428 et $1^{\text {er }}$ janvier 1429 , des 17 et 18 janvier et du 26 janvier, les Anglais s'installent dans leur système de bastilles. Mais le roman passe pudiquement sous silence le désastre du 12 février et le massacre de la journée des Harengs... où pourtant Jean de Bueil était présent. La crainte du Jouvencel est de voir arriver des renforts : «J'ai ouy dire, rapporte le messager envoyé auprès du roi, qu'il leur doit encores venir des gens que ceulx qui y sont de present, qui montent de VII à VIII mille hommes ${ }^{19}$. Ici, bien sûr, les chiffres sont ceux des estimations médiévales; rappelons que Monstrelet parlait de 10000 assiégeants, mais que le chiffre officiel de 5050 hommes, calculé d'après les comptes anglais, est, selon l'éditeur des comptes, fort peu probable : le nombre de «3 000 à la fin du siège est un maximum plutôt qu'un minimum $»^{20}$. Les renforts de l'armée anglaise amenés par petits groupes par John Fastolf n'ont sans doute jamais atteint les 1200 hommes annoncés par le Journal du Siège $e^{21}$. Mais il est certain que la fièvre obsidionale, accrue par les échecs répétés des sorties françaises, a largement contribué à surestimer le nombre des assiégeants. D'autre part, il faut se rappeler que plus la disproportion est grande entre les effectifs en présence, plus la victoire de David sur Goliath est exemplaire. Plus le Jouvencel sera «pressé» dans sa ville, plus l'intervention du comte de Parvenchères sera glorieuse.

Crathor, tout en tenant bon, ne voit plus son salut que dans l'intervention des troupes du Prince; un corps de renfort doit pouvoir entrer dans la ville : les ennemis «rompent les chemins et nous gardent nos trois portes, mais nous avons avisé ung lieu en nostre ville par où nous pouvons faire percer un trou en notre muraille pour saillir quant le secours nous venra $»^{22}$. Effectivement, malgré la présence de bastilles à l'avant de chaque porte d'Orléans, la partie nord-est de l'enceinte était relativement facile à atteindre. On envoie donc un messager, ici le «vieux maréchal», personnage regroupant tous les chefs de guerre expérimentés qui sont passés par Orléans entre octobre 1428 et avril 1429, «homme bien entendu et cognoissant en tel cas plus que nul autre (guerrier) qu' on eust peu trouver pour le temps, combien qu'ils eussent bien à besoigner de lui en la place». Rappelons que c'est le 30 janvier 1429 que le bâtard d'Orléans partit vers Blois pour tenter d'obtenir une aide véritable de la part du roi et du comte de Clermont.

${ }^{18}$ Le Jouvencel, I, p. 164-165 ; ibid., p. 168 : «Le Jouvencel a fait deux ou trois saillies ; mais à toutes les saillies, il les a foullez et reboutez ». Cette phrase ne se comprend que si, dans le second membre de phrase», « il » est l'ennemi et «les», les saillies (les sorties).

${ }^{19}$ Ibid., p. 169-170.

${ }^{20} \mathrm{~L}$. Jarry, «Le compte de l'armée anglaise au siège d'Orléans », Mémoires de la Société Archéologique et Historique de l'Orléanais, t. XXIII, p. 494.

${ }^{21} \mathrm{P}$. Charpentier et Ch. Cuissard, Le journal du siège..., Orléans, 1896, p. 26.

${ }^{22}$ Le Jouvencel, I, p. 171. 
Les entrevues du roi et du messager sont particulièrement intéressantes et doivent refléter ce que Jean de Bueil avait connu auprès de Charles VII et du dauphin Louis. Après qu'il a fait le récit de la mise en place du siège par le duc Baudoyn, le vieux maréchal est invité à sortir. Les conseillers délibèrent hors de sa présence. Les maréchaux (Jean de Brosse, Gilbert Motier), l'amiral (Louis de Culant) interviennent pour soutenir l'intervention pressante du comte de Parvenchères (Dunois et Jean d'Alençon) : il faut envoyer un renfort qui jugera sur place des occasions favorables : «les disposicions des choses donnent conseil; et ici on ne les peut savoir $»^{23}$. Le roi affirme que les gens de Crathor lui tiennent à coeur : «ils sont des meilleurs gens que j'aye ; et pour rien ne les laisseray perdre».

Mais les discussions sur la tactique à adopter sont longues et pénibles, Jean de Bueil nous livre là un exemple bien vécu des tergiversations du roi et des pressions multiples de ses familiers : on fait comparaître le messager, on l'écoute et on le fait sortir, ronger son frein pendant les conciliabules. La majorité des présents est d'avis de s'approcher de Crathor, installer à Luc (Blois) une armée et prendre la mesure de ce qui se passe ; mais le maître des arbalétriers prétend ne rien pouvoir faire avant la fin du mois (on est le 2 !). On fait revenir le vieux maréchal, le roi lui annonce qu'on fera conseil à trois heures et que, de toute façon, c'est l'heure du repas $^{24}$. Sur le point de repartir après le conseil, le vieux soldat, avec la franchise que donne la longue pratique des faits d'armes qui vous laissent remplis de gloire mais sans un sou, s'agenouille devant le souverain et lui dit: "Sire, je ne vous sauroye plus recommander la besongne, car je voi bien que vous l'avez assez à coeur [...] ; s'il me vient aucune fortune, je me recommande à vostre bonne grace. Ne vous soulciez, Mareschal, dist le Roy, je ne vous abandonneray point en tant que touche mes biens; de la vie je ne vous pourrais restituer». Et avec une assez étonnante insolence - dont il me semble qu'elle peut être à la fois ce que Jean de Bueil a pu entendre de la part des favoris royaux (on voit assez bien le connétable de Richemont ou La Trémoille faire cette sortie) et ce qu'il aurait voulu pouvoir dire à ses souverains - la répartie jaillit : «Sire, dist le Mareschal, les gens meurent, quant il plait à Dieu et la longue vie et la briefve est tant comme il lui plaist. Mais aux biens temporels il vous a donné telle puissance, la sienne mercy et grace, que vous y povez bien pourveoir. Et adieu, Sire $»^{25}$.

Le rendez-vous est pris pour le vingt-cinquième jour du mois sur la rivière, «là où se prendrait la conclusion [décision] de passer», et c'est le 24 avril 1429 que se fit l'arrivée des renforts français à Blois.

\footnotetext{
${ }^{23}$ Ibid., p. 172. Il dit aussi : «icy on ne peut conclure de telles choses; et fault qu'elles se concluent sur les lieux et selon ce qu'on treuve. Et tous ceulx qui en veullent autrement parler, faut qu'ilz devinent (qu'ils soient devins)».

${ }^{24}$ Ibid., p. 174 : «je veuil que, à trois heures après disner, vous soyez tous devers moy, affin que on baille à chascun sa charge pour faire diligence en cette matière. Or allons disner ; car j'ay ouy maistre Alinot le medecin, qui dit qu'il est temps de crier aux queulx. »

${ }^{25} \mathrm{Ibid}$., p. 176. Il y a dans l'échange un jeu sur le double sens de fortune : la richesse et le hasard (bon ou mauvais)
} 


\section{L'arrivée du comte de Parvenchères - Jeanne d'Arc}

Parvenchères, le héros de la marche victorieuse est ici le parangon des vertus militaires, le personnage syncrétique de tous les chefs de troupes de la France armagnacque, y compris la Pucelle, jamais ici évoquée. La réunion des troupes ayant eu lieu le 24, les hommes repartent quatre jours après (Jeanne quitta effectivement Blois le 28 avril). La tactique d'approche de Crathor leur est annoncée par le vieux maréchal, venu à leur rencontre ${ }^{26}:$ il faut contourner la ville, revenir par le coteau et entrer par une porte où «ceulx (des ennemis) qui sont logiez devant la porte de Bonne-Grace n'y sont seullement que pour nous tenir encloz, que nous ne puissions saillir aux champs $»^{27}$. On trouverait difficilement une meilleure définition de la bastille Saint-Loup. La réaction du comte de Parvenchères est celle de Jeanne d'Arc : on sait que Dunois a raconté sa première entrevue avec Jeanne et la colère de celle-ci quand elle vit qu'on la faisait entrer en ville, au lieu d'aller «tout droit où sont Talbot et les Anglais », et les longues explications qu'il dut donner pour accepter qu'elle attende les secours arrivant de Blois. Aux portes de Crathor, le comte s'exclame : «Nous nous attendions à les combattre dès l'arrivée » et, par un étrange jeu de miroir, Parvenchères-Jeanne s'entend répondre ce qu'avait dit DunoisParvenchères, il faut attendre le reste des troupes et le ravitaillement: «que l'on envoie hastivement Monseigneur le Prevost des Marechaux à Fleure-sur-Faire (Blois), et escrivez à tout le monde que on envoie par touz costez faire venir marchans et vivres $»^{28}$. Il ne faut pas sous-estimer l'ennemi et l'attaquer trop vite avec trop peu d'hommes ${ }^{29}$. Ainsi, seul un petit groupe accompagne le libérateur vers la ville et pour faciliter l'entrée, comme les Orléanais attaquèrent Saint-Loup, les gens de Crathor sortent et «sont desjà plus de cinq cens hors de la porte aux champs et escarmouchant de près ceulx qui se retirent».

Profitant de la diversion et dans le fracas des armes et des cloches, «tout crie, tout hue, tout bruit, trompettes sonnent, tout sonne et les cloches de la ville sonnent mesmement $»^{30}$, la troupe marche «le petit pas en moult belle ordonnance droit en la ville». Il est certain que Jean de Bueil nous rapporte ici ce qu'il vit en arrivant avec Jeanne : «il regarde par la vallée sur sa main gauche et voit le siège et ses ennemis en ordennance de bataille. Et vit clairement que tout se tiroit ensemble au champ, là où le Mareschal lui avait dit, et qu'ilz avoient tout désemparé entre lui et la ville et

${ }^{26}$ On sort et rentre de Crathor avec autant de facilité que d'Orléans : le vieux Maréchal y est revenu, puis en est ressorti avec tant d'aisance qu'il a un jour d'avance sur le rendez-vous ! À Orléans, en avril 1429, outre une sortie ratée et meurtrière le 18 , il arriva de la poudre de l'argent pour, enfin, payer la garnison et des troupes: le Bâtard de Mascaran, Giron et ses Bretons, Florent d'Illiers (Journal du siège, p. 69 à 73).

${ }^{27}$ Le Jouvencel, I, p. 182.

${ }^{28}$ Ibid., I, p. 184. Dunois, témoignant au procès en nullité, raconte qu' « il voulait aller chercher les soldats qui traversaient à Blois pour venir porter secours à ceux de cette cité mais Jeanne ne voulait guère attendre ni consentir à ce qu'il aille vers eux » (transcrit dans La libération d'Orléans, p. 226).

${ }^{29}$ " Monseigneur, il n'en fault point faire la petite bouche en leur fortiffication. Vous ne leur mesferez jà, et seroit grande follie à vous de les y assaillir », répète un peu plus tard au comte le « vieil capitaine de Crathor », Guillaume de Brézé, selon Tringant, (Le Jouvencel, I, p. 193). ${ }^{30}$ Ibid., I p. 190. 
qu'il n'y avoit plus rien ${ }^{31}$. Les ruines des abbayes de Saint-Euverte et Saint-Aignan étaient en effet le spectacle qui accueillit les arrivants d'avril 1429 et, sur la rive gauche, se dressait le camp des Augustins où l'on devait clairement voir s'agiter les ennemis avertis d'une nouvelle arrivée de renforts.

L'entrée dans la ville est «johannique», mais aussi sans doute semblable à bien d'autres entrées solennelles : les cloches et les trompettes sonnent, le cortège se dirige vers la "grant église »- la cathédrale - nommée ici Saint-Symphorien (du nom de ce faubourg de Tours ?) ${ }^{32}$, où l'on rend grâce et le comte prend ses quartiers. Là encore, des détails montrent que Jean de Bueil s'est effectivement souvenu du logement de la Pucelle et des caractères topographiques propres à Orléans. Le héros va loger «oultre» dans un faubourg séparé du camp ennemi par la rivière, mais néanmoins situé au cœur du dispositif des assiégeants. Si l'on essaye de comprendre cet étrange emplacement, on comprend très bien de quoi il s'agit. Jeanne fut logée, non pas au centre de la vieille ville, mais bel et bien hors de la muraille romaine: pour aller de la cathédrale à la maison de Jean Boucher, près de la Porte Renart, il fallait effectivement franchir une porte, sans doute démantelée, la porte Dunoise et traverser plusieurs rues ${ }^{33}$. Rappelons qu'en 1429, la rue Sainte-Catherine était encore en grande partie bordée à l'est par la muraille romaine : l'hôtel des Créneaux n'était pas construit, une porte antique et le Châtelet s'élevaient encore près de la Loire. Le «bourg-neuf» dans lequel on logea le sauveur de Crathor, Parvenchères-DunoisJeanne, ressemble bien à notre «bourg Dunois» que cinq bastilles anglaises cernaient... Mais, à la différence du héros du roman que l'on fait entrer dans une ville par une porte et sortir par l'autre, Jeanne n'eut pas besoin de fortifier elle-même sa demeure $^{34}$ ! Il est évident que Jean de Bueil, dans son travail de réorganisation du souvenir, se heurte à des incohérences dont il se sort par des astuces (pour montrer ici la rapidité avec laquelle le sauveur réussit à assurer sa sécurité dans un faubourg), mais qui ont peut-être pour origine les réflexions qu'il avait faites en son temps : pourquoi avoir dû sortir de la vieille ville, vrai «sanctuaire» au sens militaire et actuel du terme, pour aller se mettre près d'une porte, dans un quartier moins dense et plus exposé à la mitraille ennemie ?

\section{La levée du siège}

Les atermoiements des premiers jours, la dure attente, l'impatience des uns et les réticences des autres se lisent dans Le Jouvencel comme dans le Journal du siège. L'initiative individuelle, "l'émotion» populaire déplaisent à Dunois comme au comte de Parvenchères et l'épisode du 4 mai, la prise de la bastille Saint-Loup et même l'attaque des Tourelles sont devenus, dans le Jouvencel, un échec durement

\footnotetext{
${ }^{31}$ Ibid., I, p. 191.

${ }^{32}$ La cathédrale d'Orléans est dédiée à la Sainte-Croix.

${ }^{33} \mathrm{Je}$ ne me permettrais pas cependant d'avancer que la « rivière » que le comte doit passer pour arriver à son logement serait le fossé romain alors encore conservé ! Nous sommes ici dans la part de l'imaginaire romanesque.

${ }_{34}{ }$ Il (le comte) estoit homme diligent et vaillant et en peu de heure il eust fortifié son logeiz, tellement qu'il fut en seurté [...] et le lendemain il fut tellement cloz et barré qu'il n'eust plus besoing » (Le Jouvencel, t. I, p. 194).
} 
stigmatisé $^{35}$. «Le conte avoit deffendu que nul ne allast droit au pont». Aussi la sentence est-elle sèche : «Les folz font les folies. Qui a les coups si les boyve ». Afin d'atténuer cette condamnation et expliquer ces folles sorties, le Jouvencel lui-même a pourtant cette belle phrase : «et noz genz ont commencié ceste folie pour le couraige qu'ilz ont de vostre venue».

Ici se place le grand hiatus : il n'y a pas de combat victorieux, pas de pont repris, pas de blessure du héros, pas de 7 mai en somme. L'action reprend quand des espions viennent dans la nuit annoncer : «Le duc de Baudoyn seurement desloge. Comment, dist le conte, il n'est pas encore jour! Je ne croy pas que ung tel homme parte avant le jour : ce ne seroit pas honneste chose à lui $»^{36}$. Et là, on croirait bien que Jean de Bueil a lu la Chronique de la Pucelle : "Les Anglais tinrent cette nuict grand conseil $[\ldots]$; si issirent le dimanche huictieme jour de mai et s'en allèrent en belle ordonnance, leurs étendards desployés », écrit le chroniqueur. "Ainsi marcha le duc Baudoyn et les siens en belle ordonnance, pas pour pas », reprend notre roman.

La réaction des chefs de Crathor et d'Orléans fut la même : on ne poursuit pas les troupes qui se retirent: la Pucelle «si fist apporter une table et un marbre et dire deux messes ${ }^{37}$. Le comte de Parvenchères, pour sa part, «manda quérir chascun et s'en alla au lieu où la messe estoit appareillée, qui estoit en ung carrefour, au large, au pié d'une croix, affin que chascun la ouyst $\gg^{38}$. La ville est libérée, elle rend grâce. Toute victoire se terminait ainsi.

\section{Le compagnon sans mémoire}

Il faut bien dire que le silence qui couvre les faits et gestes de la Pucelle, dans le roman à grosses clefs de Jean de Bueil, n'est pas sans poser quelques problèmes. Le commentateur Guillaume Tringant l'a bien senti, qui, sans donner d'explication, ni même sans relever le fait que Jeanne n'est pas présente dans le roman, consacre plusieurs pages à raconter le rôle que joua la jeune fille, «gardant les bestes » puis devenue "une chose de la grâce de Dieu », à Chinon, Orléans, Paris, Compiègne. Mais de la suite, prise, procès et bûcher, chez lui non plus, il n'est pas question ${ }^{39}$.

${ }^{35}$ Ibid., I., p. 196. Cette dureté est, dans le cadre d'un roman qui est un traité d'éducation militaire, considérée comme parfaitement pédagogique. L'excuse présentée par le Jouvencel est repoussée par le comte : "Or çà il ne faut plus ramentevoir (revenir sur) des choses passées, sinon chastier ceulx qui ont commencié la folye».

${ }^{36}$ Ibid., p. 206. C'est en fait ce qui s'est passé à Lagny quand le duc de Bedford, n'ayant pu empêcher une partie des troupes royales d'entrer dans la ville, apprit qu'une autre colonne arrivait depuis la Ferté-sous-Jouarre ; il craignit une attaque sur Paris et «leva son siège moult hastivement et sans ordonnance, et laissa là ses bombardes, canons et autre artillerie » (Jean Chartier, Chronique, t. I. p. 146). En revanche, cette précipitation n'est pas le bon ordre que l'on trouve à Orléans et dans le roman du Jouvencel.

${ }^{37}$ Chronique de la Pucelle, t. I, p. 296.

${ }^{38}$ Le Jouvencel, t. I, p. 208.

${ }^{39}$ Le Jouvencel, Commentaire de Tringant, t. II, p. 277 à 282. Guillaume Tringant abandonne Jeanne après l'échec devant Saint-Denis : «Mais le bon roy Charles ne put plus tenir son armée ensemble par faute de vivres, et s'en revint le Roy en Touraine, et laissa la pucelle es pays qu'ilz avoient conquis, accompagnée de beaucoup de gens de biens et bons capitaines ». L'amiral absout le roi avant tout reproche. 
Il semble sûr que c'est de volonté délibérée que Jean de Bueil n'a pas voulu compter Jeanne au nombre des modèles à offrir à un futur chef de guerre. D'abord il a pu hésiter à rappeler que le rôle bénéfique de la jeune fille ne fut récompensé que par beaucoup d'ingratitude et surtout d'incompréhension de la part du souverain ${ }^{40}$. Et dans un temps où le vieil amiral était en opposition avec le fils de Charles VII, il se révélait plus politique de rappeler les lumières que les ombres du règne du père. Il fallait excuser les réserves de Jean de Bueil envers Louis XI en embellissant son prédécesseur.

Ensuite, et surtout, il ne faut pas oublier que Le Jouvencel, plus qu'un roman, est une œuvre didactique : il s'agit de montrer toutes les qualités nécessaires au bon meneur d'hommes, au conseiller avisé, au futur amiral. Or Jeanne d'Arc n'est évidemment en rien un bon modèle. Un vrai capitaine est élevé pour cela, la guerre est un métier - et même "un plaisant métier et bon à jeunes gens $»^{41}-$ et toutes les réformes royales furent tendues vers ce but: on ne s'improvise donc pas chef de troupe et stratège. Le Jouvencel a souffert du froid, de la faim et de la peur dans des forteresses minables, avançant petitement, commençant par se mettre à l'école d'un capitaine puis d'un autre, passant tous les degrés de la carrière, du page obscur à la lieutenance royale, du vol de linge pour bourrer sa «jacque » au mariage avec la fille d'un roi. Peu à peu, il a acquis une vaillance et une science à l'antique et, quand il s'agit de l'art de prendre les bastilles ou d'attaquer une ville, ce n'est pas de folie qu'il est question mais de poliorcétique, avec Végèce à l'appui ${ }^{42}$. Jamais Jeanne ne serait devenue Penthésilée; le Jouvencel, lui, n'est pas loin de se prendre pour Alexandre.

La guerre demande à la fois des prévisions à long terme - un capitaine «doit prévenir avant la main ad ce qui lui peut advenir $»^{43}-$, de la souplesse d'esprit, une adaptation aux conditions de l'instant et des capacités de dissimulation. Or ce portrait est incompatible avec l'image têtue et si droite de la fille de Domrémy.

La guerre est une affaire de spécialistes et l'épopée de Jeanne est aberrante, sa fulgurance est émouvante et elle fut pleinement efficace en un moment crucial.

${ }^{40}$ G. Peyronnet, «Jeanne d'Arc et Charles VII : un problème à revoir », Bulletin de l'Association des amis du Centre Jeanne d'Arc, $\mathrm{n}^{\circ} 12,1988$, p. 7-23.

${ }^{41}$ « C'est joyeuse chose que la guerre; on y ouit, on y voit beaucoup de bonnes choses, et y apprent moult de bien. Quant elle est en bonne querelle, c'est justice, c'est défendre droiture ». Après la libération de la ville de Crathor, le Jouvencel fait cette profession de foi en faveur de la guerre juste et, surtout, de la camaraderie militaire : "on s'entr'ayme tant à la guerre » (Le Jouvencel, t. II, p. 20).

${ }^{42}$ Le Jouvencel, t. II, chapitre XVI, p. 31 et suivantes. Dans un long discours, le sire de Chamblay explique au Jouvencel qu'assiéger une ville en construisant des bastilles n'a pas réussi aux Anglais : ils se sont épuisés à défendre ces points que les Français ont pris les uns après les autres : «les ay-je veu prendre et desconfire devant Orléans, devant Compiègne, devant Dieppe et devant le Mont-Saint-Michel ; et je crois qu'elles ont plus profité, quelque part qu'elles ayent esté mises, aux ennemys qu'à ceulx à qui elles estoient». Le paragraphe qui suit s'intitule : «S'ensuit l'opinion de Végèce pour assiéger une forte ville». Il s'agit en fait d'une très libre interprétation de l'antique où il est affirmé la nécessité de posséder 248 pièces d'artillerie, avec poudre et charbon (sur le rôle des manuels d'art militaire, cf. Ph. Contamine, La guerre au Moyen Âge, Paris, $2^{\mathrm{e}}$ éd., 1986, p. 353-364).

${ }^{43}$ Le Jouvencel, t. II, p. 259 ; c'est un extrait de la conclusion de l'ouvrage. 
En aucun cas elle n'est pédagogique. C'est un modèle à ne pas suivre. Voilà l'argument fondamental : Jean de Bueil ne pouvait pas passer sous silence l'épisode d'Orléans : c'était une suite d'exemples magnifiques de problèmes militaires en cas de siège et de la tactique à adopter pour dialoguer avec le roi, détenteur des finances et du droit de lever les troupes. En aucun cas, néanmoins, la libération ne devait participer du miracle : un chef de guerre, si confiant en la valeur de sa juste cause et la protection divine soit-il, ne peut laisser entrer dans ses calculs la moindre part de merveilleux, et l'expérience avait d'ailleurs prouvé que les miracles ne se renouvellent pas. L'entêtement de Jeanne était certainement, aux yeux de Jean de Bueil, la cause de son échec personnel.

Ce qu'il voulait montrer dans son récit était une carrière réussie, la sienne. Pour ne pas raconter le revirement de fortune de celle qui avait mené le roi à Reims, le compagnon infidèle a préféré ne pas même parler de ses moments glorieux. On peut alors se demander s'il ne fut pas de ces esprits positifs et froids qui n'avaient accepté que par contrainte morale et fidélité dynastique la lubie royale qui avait un moment permis l'intrusion d'une femme illuminée et inexperte dans un compagnonnage de rudes hommes rompus au métier de la guerre. 